\title{
COLOR IMAGE RETRIEVAL BASED ON NON-PARAMETRIC STATISTICAL TESTS OF HYPOTHESIS
}

\author{
R. Shekhar ${ }^{1}$ and K. Seetharaman ${ }^{2}$ \\ ${ }^{1}$ Department of Computer Science \& Engg., Research scholar, \\ Manonamainam Sundaranar University, Tirunelveli-627012, INDIA, \\ ${ }^{2}$ Department of Computer Science \& Engineering, \\ Annamalai University, Annamalai Nagar, INDIA, \\ kseethaddeau@gmail. com
}

\begin{abstract}
A novel method for color image retrieval, based on statistical non-parametric tests such as twosample Wald Test for equality of variance and Man-Whitney U test, is proposed in this paper. The proposed method tests the deviation, i.e. distance in terms of variance between the query and target images; if the images pass the test, then it is proceeded to test the spectrum of energy, i.e. distance between the mean values of the two images; otherwise, the test is dropped. If the query and target images pass the tests then it is inferred that the two images belong to the same class, i.e. both the images are same; otherwise, it is assumed that the images belong to different classes, i.e. both images are different. The proposed method is robust for scaling and rotation, since it adjusts itself and treats either the query image or the target image is the sample of other.
\end{abstract}

\section{KEYWORDS}

variance, mean, query image, target image, non-parametric tests..

\section{INTRODUCTION}

In recent years, a number of researchers have turned their attention to the content-based image retrieval (CBIR) system. In CBIR system, the researchers concentrate on developing low-level global visual features, namely color properties, shape, texture, and spatial relationship etc., which are used as query for the retrieval process [1-4]. The method proposed in [5-10; 10-15] classifies or segments the entire image into various regions according to the objects or structures present in the image, and the region-to-region comparison is made to measure the similarity between two images [3,11,12]. In a region-based system, the user has to provide one or more regions from the query image to start a query session. Automatic and precise extraction of image objects is still beyond the ability of the retrieval system available with the computer vision [13]. Therefore, the above system tends to partition one object into several regions, but none of them is representative of the semantic object.

Content-based image retrieval system gives results with low accuracy and slow response time, because there is a big gap between semantic concepts and low-level image features [14]. A

David C. Wyld et al. (Eds) : COSIT, DMIN, SIGL, CYBI, NMCT, AIAPP - 2014

pp. 131-138, 2014. (C) CS \& IT-CSCP 2014

DOI : $10.5121 /$ csit.2014.4912 
concept, relevance feedback, has been developed to bridge the gap [15-18]. In [14], a new relevance feedback approach is proposed, which uses Bayesian classifier and treats positive and negative feedback images with different strategies. In relevance feedback method, the user has to provide positive and negative feedback images to improve the performance of the system. Minka and Picard [18] propose the FourEyes system, which has two disadvantages: (i) it uses the regionto-region similarity measure; (ii) the re-clustering of all the features when a new image is added. Thus, it is not very scalable [5]. To overcome this, Jing et al. [5] proposed a system with the features: (i) it computes probabilistic interpretation and it is used in region matching; (ii) region codebook is used; (iii) the SVM based classifier and clustering techniques are adopted, but it requires high computational effort. Above all these, it requires positive and negative query image examples.

Theoharatos et al. [23] proposed a system, based on multivariate non-parametric test, namely Wald-Wolfowitz test (WW-test), and graph theoretic framework of minimal-spanning-tree (MST). In this work, first, the MST is constructed based on the sample identities of the points taken from the images. Based on the consecutive sequence of identical sample identities, runs of the sample points are computed and the WW-test is employed to identify whether the query and target images are same or not. In this work, the drawbacks are

(i) Construction of the MST demands computational overhead.

(ii) Based on the sample identities of the points, run length of each sample identical identities is computed and then the WW-test is used to identify whether the query and target images are same or not.

In this paper, a unified technique is proposed for automatic image retrieval, based on nonparametric tests such as two-sample Wald Test for equality of variance and Man-Whitney U test. In the proposed technique, mean and variance (first and second moments of the sample points) are used as representatives of both query and target images. The methods proposed in $[19,20]$ retrieve only the texture images with intensity values ranging from $0-255$, i.e. gray-scale images. This motivated us to develop a new method which retrieves color images; both texture and structure images; and invariant for rotation and scaling.

\section{PROPOSED TEST STATISTIC FOR SiMILARITY OF IMAGES}

Let $\mathrm{x}$ be a random variable that represents the intensity value with additive noise of a pixel at location $(k, l)$ in a color image. The pixel $\mathrm{X}(k, l) \in \mathfrak{R}^{3}$ is a linear combination of three colors such as red, green and blue, i.e. $\mathrm{X}(k, l)=[\mathrm{r}(k, l), \mathrm{g}(k, l), \mathrm{b}(k, l)]^{\mathrm{T}}$, where $\mathrm{T}$ represents the transformation of the vector.

\subsection{TEST FOR VARIATION BETWEEN THE QUERY AND TARGET IMAGES}

Either the query image or the target image is treated as sample while the other is treated as population. To test whether the two images are same or not, first, the variation among the intensity values of the two images are tested, i.e. $H_{0}: \sigma_{q}=\sigma_{t}$, where $\sigma_{q}$ and $\sigma_{t}$ represent the variation among the intensity values of the query and target images respectively. To achieve this, the test for homogeneity of variances is employed, i.e. two-sample Wald Test for equality of variance [22]. The $R$ test is nearly as robust as Levene's test and nearly as powerful as the $F$ test. Hypotheses:

$$
\mathrm{H}_{0}: \sigma_{\mathrm{q}}=\sigma_{\mathrm{t}} \text { (Similarity }- \text { query and target images belong to the same class) }
$$


$\mathrm{H}_{\mathrm{a}}: \sigma_{\mathrm{q}} \neq \sigma_{\mathrm{t}}$ (Non-similarity - query and target images belong to different class)

Test Statistic: The two-sample Wald test statistic [22] defined in equation (1) is applied to test wheteher the images are same or not.

$$
R=\frac{\left(S_{q}^{2}-S_{t}^{2}\right)^{2}}{\left(\left(m_{q 4}-S_{q}^{4}\right) / n_{q}+\left(m_{t 4}-S_{t}^{4}\right) / n_{t}\right)}
$$

where, $\mathrm{S}_{\mathrm{i}}^{4}=\left(\mathrm{m}_{4}-\mathrm{m}_{\mathrm{t}}^{2}\right) / \mathrm{n} ; \mathrm{S}^{2}$ is the unbiased sample variance; $\mathrm{m}_{\mathrm{i} 4}$ are the fourth central sample moments for the $\mathrm{i}$-th sample and $\mathrm{i}=\mathrm{q}, \mathrm{t} ; \mathrm{n}_{\mathrm{q}}$ and $\mathrm{n}_{\mathrm{t}}$ are the number of pixels in the query and target images respectively.

The query and target images are judged to be same, if $\mathrm{R}<\mathrm{c}_{\alpha}$, where, $\mathrm{c}_{\alpha}$ is the point for which the Chi-square distribution has weight $\alpha$ in the right hand tail, then the $R$ test rejects $\mathrm{H}_{\mathrm{o}}$ at approximately $100 \alpha \%$ level; otherwise, the two images are assumed to be different.

\section{2 . TEST STATISTIC FOR EQUALITY OF SPECTRUM OF ENERGY BETWEEN THE IMAGES}

As discussed in the previous section, if the variation among the intensity values of the query and target images passed the test for homogeneity of variances, then it is proceeded to test the equality of means of the two images. To achieve this, the test for equality of means, i.e. Mann-Whitney $U$ (MWU) test [23] is employed. In general, the actual pixel values either in the query image or the target image may exeed 20, thus value of $U$ approaches Gaussian distribution, and thus the null hypothesis can be tested by $\mathrm{Z}$ test. The MWU test is a greater powerful than the t-test if the populations are non-normal distributions, i.e. a mixture of normal distributions, and it is also nearly as efficient as the t-test in the case of Gaussian distributions.

The test of hypothesis are assumed as follows.

Hypotheses:

$\mathrm{H}_{0}$ : The query and target images are belonging to the same class.

$\mathrm{H}_{\mathrm{a}}$ : The query and target images are belonging to different class.

The test statistic $(Z)$ is defined as in equation (2),

$$
\mathrm{Z}=\frac{\mathrm{U}-\mathrm{E}(\mathrm{U})}{\mathrm{SD}_{\mathrm{UCorr}}}
$$

where,

$$
\mathrm{U}=\left(\mathrm{n}_{\mathrm{q}} \times \mathrm{n}_{\mathrm{t}}\right)+\frac{\mathrm{n}_{\mathrm{q}} \times\left(\mathrm{n}_{\mathrm{q}}+1\right)}{2}-\mathrm{T}_{\mathrm{q}}
$$

$\mathrm{T}_{\mathrm{q}}$ is the larger of the sum of ranks of either the query image or the target image; $\mathrm{n}_{\mathrm{q}}$ and $\mathrm{n}_{\mathrm{t}}$ are the number of pixels in the query and target images respectively.

$$
\mathrm{E}(\mathrm{U})=\frac{\mathrm{n}_{\mathrm{q}} \times \mathrm{n}_{\mathrm{t}}}{2}
$$




$$
\mathrm{SD}_{\mathrm{UCorr}}=\sqrt{\frac{\mathrm{n}_{\mathrm{q}} \times \mathrm{n}_{\mathrm{t}}}{\mathrm{n}(\mathrm{n}-1)}\left(\frac{\mathrm{n}^{3}-\mathrm{n}}{12}-\sum_{\mathrm{i}=1}^{\mathrm{k}} \frac{\mathrm{t}_{\mathrm{i}}^{3}-\mathrm{t}_{\mathrm{i}}}{12}\right)} \text {, and } k \text { is number of tied ranks; } \mathrm{t}_{\mathrm{i}} \text { is the }
$$

number of subjects sharing rank $i$.

$$
\begin{aligned}
& \mathrm{n}=\mathrm{n}_{\mathrm{q}}+\mathrm{n}_{\mathrm{t}} \\
& \mathrm{U}_{\mathrm{q}}=\left(\mathrm{n}_{\mathrm{q}} \times \mathrm{n}_{\mathrm{t}}\right) \times \frac{\mathrm{n}_{\mathrm{q}} \times\left(\mathrm{n}_{\mathrm{q}}+1\right)}{2}-\mathrm{T}_{\mathrm{q}} \\
& \mathrm{U}_{\mathrm{t}}=\left(\mathrm{n}_{\mathrm{q}} \times \mathrm{n}_{\mathrm{t}}\right)-\mathrm{U}_{\mathrm{q}} \\
& \mathrm{n}=\mathrm{n}_{\mathrm{q}}+\mathrm{n}_{\mathrm{t}}
\end{aligned}
$$

Critical region: It is concluded that the query and target images are same, if $\mathrm{Z}<\mathrm{Z}_{\mathrm{C}_{\alpha}}$, where $\mathrm{Z}_{\mathrm{C}_{\alpha}}$ is the critical value at the level of significance $\alpha$; otherwise, it is concluded that the images are different.

\section{IMAGE FEATURE DATABASE CONSTRUCTION AND INDEXING}

An image database is constructed using various types of images collected from standard Brodatz album, Vistex, and Corel image databases, and from other sources such as internet and images captured by digital camera.

The feature vectors of the query image are matched with the features in the feature vector database using the test statistic discussed in the previous section. The target images are selected from image database based on the significance level $(\alpha)$ at $20 \%$. The significance level can be fixed by the user at $1 \%$ or $5 \%$ or $10 \%$ or $15 \%$ or $20 \%$ or at any other level according to the user's requirements. The selected images are indexed (ranked) according to the test statistic values, and the distance value between the query and target images from lowest to highest, i.e. in ascending order. The user can select the top most images from the indexed list according to his requirements.

\section{Measure of Performance}

In order to measure the performance of the proposed method, the precision and recall measures [25] are used, which are given in equations (3) and (4).

$$
\begin{aligned}
& \text { Pr ecision }=\frac{\mid\{\text { Re levant Images }\} \text { I }\{\text { Re trieved Images }\} \mid}{\mid \text { Re trieved Images } \mid} \\
& \text { (3) } \\
& \text { Recall }=\frac{\mid\{\text { Re levant Images }\} \text { I }\{\text { Re trieved Images }\} \mid}{\mid \text { Relevant Images } \mid}
\end{aligned}
$$

\section{IMAGE DATABASE DESIGN AND EXPERIMENTAL RESULTS}

In order to implement the proposed method, 477 color images of size $512 \times 512$ pixels have been collected from various sources, i.e. 152 texture images from Brodatz Album, 176 images from Corel image database and 149 images from VisTex image database. The remaining 58 images with size $128 \times 128$ are photographed with digital camera; 43 images with size $128 \times 128$ have been 
downloaded from internet [30]. The textured images collected from Brodatz, Coral and VisTex image databases are divided into 16 non-overlapping sub-images of size $128 \times 128$. To examine the proposed system is invariant for rotation and scaling; the images are rotated by $90^{\circ}, 180^{\circ}$ and $270^{\circ}$, and scaled.

To validate the proposed system, based on statistical non-parametric tests, the concepts discussed in sections 2.1 and 2.2 are implemented with the image database constructed as discussed above. Due to space constraints, for sample, some of the images considered for the experiment are presented in Figures 1 and 2. The experiment is conducted at various levels of significance for the input query image given in column 1 of Figure 1. The images in columns 2, 3, 4, 5 of Figure 1 are retrieved at level of significance, 0.001; images in columns 6, 7 are retrieved at 0.05 level of significance; at 0.15 level of significance, the system retrieves the images in columns 8, 9, 10 and 11.

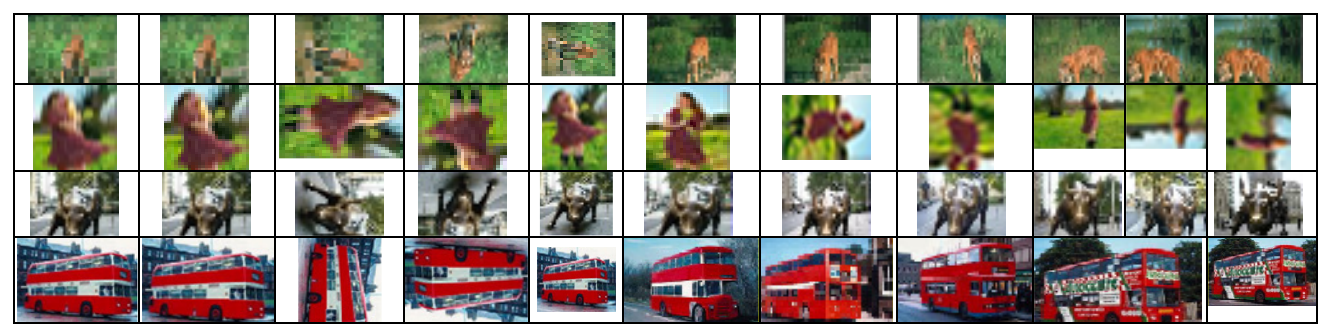

Figure 1. Structure images; column 1: input query image; columns 2 - 11: retrieved output images

Furthermore to emphasize the efficiency of the proposed method, the images in column 1 of Figure 2 are given as input query to the system. The images in columns 2, 3, 4, 5 of Figure 1 are retrieved at level of significance, 0.001; images in columns 6, 7 are retrieved with level of significance at 0.03 ; significance level at 0.12 , the system retrieves the images in columns $8,9,10$ and 11 .

The obtained results emphasize that the proposed system is robust for scaling and rotation, since it retrieves the same input query image rotated by 90 degrees, 180 degrees, 270 degrees, and scaled images at the level of significance 0.001 .

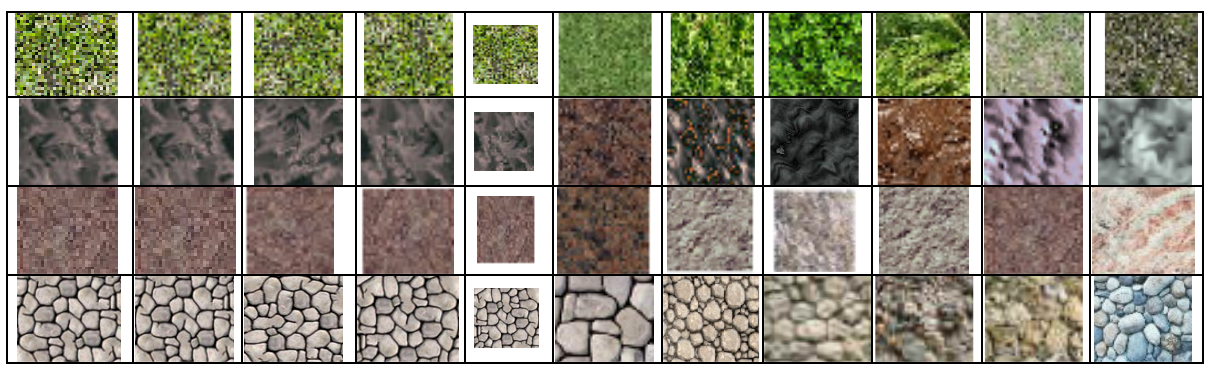

Figure 2. Texture images; column 1: input query image;columns $2-11$ : retrieved output images 
Table 1. Performance measure of the proposed method with other existing methods

\begin{tabular}{|c|c|c|c|c|c|c|c|c|c|c|}
\hline \multirow[t]{2}{*}{ Database } & \multicolumn{2}{|c|}{$\begin{array}{l}\text { Proposed } \\
\text { method }\end{array}$} & \multicolumn{2}{|c|}{$\begin{array}{c}\text { Statistical } \\
\text { Distributional } \\
\text { Approach } \\
\end{array}$} & \multicolumn{2}{|c|}{$\begin{array}{c}\text { Orthogonal } \\
\text { polynomial }\end{array}$} & \multicolumn{2}{|c|}{ Gabor wavelet } & \multicolumn{2}{|c|}{$\begin{array}{l}\text { Contourlet } \\
\text { Transform }\end{array}$} \\
\hline & Recall & Precision & Recall & Precision & Recall & Precision & Recall & \begin{tabular}{|l|} 
Precision \\
\end{tabular} & Recall & Precision \\
\hline Brodatz & 0.840 & 0.781 & 0.836 & 0.784 & 0.812 & 0.685 & 0.824 & 0.661 & 0.825 & 0.601 \\
\hline VisTex & 0.822 & 0.799 & 0.810 & 0.795 & 0.782 & 0.681 & 0.795 & 0.670 & 0.805 & 0.610 \\
\hline $\begin{array}{l}\text { Structure } \\
\text { Images }\end{array}$ & 0.850 & 0.824 & 0.842 & 0.815 & -- & -- & -- & -- & 0.826 & 0.621 \\
\hline
\end{tabular}

A comparative study is performed with the existing methods such as Orthogonal polynomial [19], Gabor wavelet transform [20] and the Contourlet transform [27] methods and Statistical distributional approach [28], and the obtained results are presented in Table 1. The results reveal that the proposed method outperforms the existing methods. It is observed from the results that there is no significant difference between the proposed method and the statistical distributional approach. Though both parametric and non-parametric tests yield almost same results; there are some difficulties to employ parametric tests. Since some type of images may not be distributed to Gaussian, at that situation the parametric tests cannot be applied. Thus, the necessity arises to use appropriate non-parametric tests instead of parametric tests.

\section{DISCUSSIONS AND CONCLUSION}

Block-wise sampling technique proposed in [21] does not yield good results for the rotated and scaled images, because the corresponding blocks of query (actual) and target (transformed) images do not match spatially, while the target image is rotated or scaled. Hence, the technique proposed in [21] fails to match and retrieve the right images. The proposed system avoids this problem, because it uses the global distributional differences of both query and target images; in the case of structured images, these features are extracted from the shapes in both query and target images, and those are compared shape-wise, it compares the number of shapes between the images. The orthogonal polynomial based method [19] retrieve only textured images with grayscale, and the Gabor features based method [20] retrieves only the textured images in both color and gray-scale. The proposed system retrieves both textured and structured color images, and it is robust for scaled and rotated images. Most of the existing methods retrieve a set of similar images, from which the user has to select the required images. But the proposed system facilitates the user to retrieve the required image only by fixing the level of significance at a desired level.

In this paper, a unified system for both structured and textured color image retrieval is used, based on statistical non-parametric tests of hypothesis, namely test for equality of variances variation between the query and target images, and the test for equality of means - spectrum of energy. The proposed system is invariant for rotation and scaling, since the query image is treated as either a sample or population of the target image. First, the test for equality of variation between the query and target images is performed; the query and target images pass the test, viz. the two images are same or similar, then the test for equality of mean vectors is performed, i.e. testing the spectrum of energy on the same images. If the query and target images pass these two tests, it is inferred that the two images are identical; otherwise, it is assumed that the images belong to different groups. The proposed system provides hundred percent accuracy and precision, even if either the target or query image is rotated or scaled. 


\section{REFERENCES}

[1] J.Huang, S.R. Kunar, M.Mitra, W.J. Zhu, and R.Zabih, Image indexing using color correlogram, in: Proc. IEEE Comp. Soc. Conf. Comp. Vis. and Pattern Recognition, vol. 1, 1997, pp. 762-768.

[2] M.Stricker and M. Orengo, Similarity of color images, in: Storage and Retrieval for Image and Video Databases, Proc. SPIE 2420, vol. 1, 1995, pp. 381-392.

[3] A.Pentland, R.Picard, and S. Sclaroff, Photobook: Content-based manipulation of image databases, International Journal of Computer Vision 18(3) (1996) 233-254.

[4] Chiou-Shaan Fuh, Shun-Wen Cho, and Kai Essig, Hierarchical color image region segmentation for content-based image retrieval system, IEEE Transactions on Image Processing 9(1) (2000) 156-162.

[5] F. Jing, M. Li, H.J. Zhang, and B.Zhang, An efficient and effective region-based image retrieval framework, IEEE Transactions on Image Processing 13(5) (2004) 699-709.

[6] Jun-Wei Hsieh and W.Eric L. Grimson, Spatial template extraction for image retrieval by region matching, IEEE Transactions on Image Processing 12(11) (2003) 1404-1415.

[7] S. Belongie, C.Carson, H.Greenspan, and J.Malik, Recognition of images in large databases using color and texture, IEEE Transactions on Pattern Analysis and Machine Intelligence 24(8) (2002) 1026-1038.

[8] F. Jing, B.Zhang, F.Z.Lin, W.Y.Ma, and H.J.Zhang, A novel region-based image retrieval method using relevance feedback, in: Proc. 3rd ACM Int. Workshop on Multimedia Information Retrieval (MIR), 2001.

[9] Y.Deng and B.S.Manjunath, (1999) "An efficient low-dimensional color indexing scheme for regionbased image retrieval, in: Proc. IEEE Int. Conf. ASSP”, Vol.6, pp. 3017-3020.

[10] Ing-Sheen Hsieh and Huo-Chin Fan, (2001) "Multiple classifiers for color flag and trademark image retrieval”, IEEE Transactions on Image Processing, Vol. 10, No.6, pp. 938-950.

[11] J.R. Smith and C.S. Li, (1999), "Image classification and querying using composite region templates, Journal of Computer Vision and Image Understanding”, Vol. 75, No. 12, pp. 165-174.

[12] J.Z. Wang and Y.P. Du, (2001) "Scalable integrated region-based image retrieval using IRM and statistical clustering", in: Proc. ACM and IEEE Joint Conference on Digital Libraries, VA, 2001, pp. 268-277.

[13] J.Z. Wang, J. Li, and G.Wiederhold, (2001) "SIMPLIcity: Semantics-sensitive integrated matching for picture libraries", IEEE Transactions of Pattern Analysis and Machine Intelligence, Vol. 23, No. 9, pp. 947-963.

[14] Z. Su, H. Zhang, S.Li, and S. Ma, (2003) "Relevance feedback in content-based image retrieval: Bayesian framework, feature subspaces, and progressive learning", IEEE Transactions on Image Processing, Vol. 12, No. 8, pp. 924-937.

[15] I.J.Cox, T.P.Minka, T.V.Papathomas, and P.N.Yianilos, (2000) “The Bayesian image retieval system, pichunter: Theory, implementation, and psychophysical experiments", IEEE Transactions on Image Processing, Vol. 9, No. 1, pp. 20-37.

[16] Y.Rui and T.S.Huang, (1999) "Relevance feedback: A power tool for interactive content-based image retrieval”, IEEE Circuits Syst. Video Technology, Vol. 8, No. 5, pp. 644-655.

[17] N.Vasconcelos and A.Lippman, (1999) "Learning from user feedback in image retrieval systems", in: Proc. NIPS'99, Denver, CO.

[18] T.P. Minka and R.W. Picard, (1997) "Interactive learning using a society of models", Pattern Recognition, Vol. 30, No. 4, pp. 565-581.

[19] R. Krishnamoorthy, S. Sathiya devi, (2012) "A multiresolution approach for rotation invariant texture image retrieval with orthogonal polynomial model”, Journal of Visual Communication and Image Representation, Vol. 23, No. 1, pp. 18-30.

[20] Ju Han, Kai-Kuang Ma, (2007) "Rotation invariant and scale invariant Gabor features for texture image retrieval", Image and Vision Computing, V 25, No. 9, pp. 1474-1481.

[21] Huang, C.W., Lin, K.P., Hung, K.C. (2014) "Intuitionistic fuzzy c-means clustering algorithm with neighborhood attraction in segmenting medical image", Soft Computing, DOI: 10.1007/s00500-0141264-2.

[22] Allingham, D., Rayner, J. C. W., (2011) "A Nonparametric Two-Sample Wald Test of Equality of Variances", Advances in Decision Sciences, doi:10.1155/2011/748580.

[23] C. Theoharatos, N.A. Laskaris, G. Economou, and S. Fotopoulos (2005) "A Generic scheme for color image retrieval based on the multivariate Wald-Wolfowitz test", IEEE Transactions on Knowledge and Data Engineering, Vol.17, No. 6, pp. 808-820. 
[24] M. Kokare, B.N. Chatterji, P.K. Biswas (2003) "Comparison of similarity metrics for texture image retrieval, in: IEEE Proceedings on TENCON 2003 Conference on Convergent Technologies for AsiaPacific Region, Vol. 2, pp. 571-575.

[25] Powers, David M. W. (2012), "The Problem with Kappa, Proceedings of the 13th Conference of the European Chapter of the Association for Computational Linguistics (EACL2012) Joint ROBUSUNSUP Workshop, pp. 345-355.

[26] http://elib.cs.berkley.edu/

[27] Ch. Srinivasa Rao, S. Srinivas kumar, B.N. Chatterji, (2007) "Content based image retrieval using Contourlet Transform", ICGST-GVIP Journal, Vol. 7, No. 3, pp. 9-15.

[28] K. Seetharaman, M. Jeyakarthic, (2014), "Statistical distributional approach for scale and rotation invariant color image retrieval using multivariate parametric tests and orthogonality condition", Journal of Visual Communication Image Representation, Vol. 25, No. 5, pp. 727-739.

\section{AUTHORS}

R. Shekhar has vast experience in teaching subjects related to Computer Science \& Engineering. He has a post-graduate degree in Computer Networks and is currently pursuing doctoral studies at the MS University; Tirunelveli.He is serving as an Editorial member of IJITCS, an International Journal and has been a reviewer for several National and International Journals. He is Also a Sitting member of different National and international Associations in the field of Computer Science. His Publications appear in many Journals of National and International repute. His researches interests include image processing, Computer Networks and Artificial Intelligence.

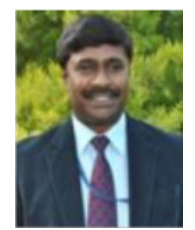

Dr. K.Seetharaman received his M.Sc. degree in Statistics from Annamalai University, Chidambaram, India in 1990 and the M.S. degree in Software Systems from Birla Institute of Technology and Science, Pilani, India in 1995. He received his Ph.D. degree in Computer Science \& Engg. from Annamalai University in 2006.He is currently working as Associate Professor in the Department of Computer Science \& Engg., Annamalai University. His research interests include Statistical Pattern Recognition,

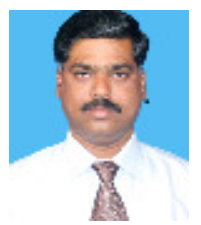
Scene Analysis, Digital Image Analysis, and Data Mining and Knowledge Discovery. He is also actively involved in professional activities, viz. reviewer in journals: Pattern Recognition, Knowledgebased Systems, IEEE Transactions on Pattern Analysis and Machine Intelligence, He is currently serving as Associate Editor of the International Journal - Aloy Journal of Soft Computing and Applications, and the Editorial Board Member of the International Journal of Information Technology and Management Sciences. $\mathrm{He}$ is also the member of the professional societies - IEEE, ACM, IAENG, and Indian Science Congress Association. 